27.-30.9.2012 in Prag, CZ 21. EADV Congress - Skin is vital

Veranstalter:

\section{EADV}

Information:

EADV Prague 2012 c/o CZECH-

IN s.r.o.

Tel: $+420 / 261 / 174301$

Fax: +420/261/174 307

E-Mail: info@eadvprague2012.

org

28.-29.09.2012, Meran/Tirol

17. Jahrestagung der Österreichischen Gesellschaft für Dermatochirurgie

Leitung

Prof. Dr. K. Böhler, Dr. Simon

Gamper, Dr. Josef Koller

Information

Ilona Fuchs Congress

Tel: 0043/(0)1-6022548

Fax: 0043/(0)1-6022548-90

E-Mail: office@ifc.co.at

24.11.2012, Dresden

Neues aus Diagnostik und

Therapie; Tagung der Säch-

sischen Gesellschaft für

Dermatologie e.V.

Leitung

Prof. Dr. med. Uwe Wollina

Tagungssekretär

Dr. med. Andre Koch

Information:

Chefarztsekretariat Annette

Thiele

Tel: 0351/480-1685

Fax: 0351/480-1219

E-Mail:Thiele-an@khdf.de

24.11.2012, Lübeck

3. Lübecker Allergie-

Symposium

Veranstalter

UKSH, Campus Lübeck, Klinik

für Dermatologie, Allergologie und Venerologie

Information

Prof. Dr. Uta Jappe, Wiebke

Schmidt-Tomkewicz ( $\mathrm{Di}+\mathrm{Fr}$ )

Tel: 0451/5006985

Fax: 0451/5002981

E-Mail: wiebke.schmidt-tomkewicz@uksh.de

\title{
Versorgungsforschung für die politische Kommunikation unverzichtbar
}

In den vergangenen dreizehn Jahren gab es zehn Gesundheitsreformen nicht unbedingt zum Vorteil für Ärzte und Patienten. Ein professioneller Dialog mit Entscheidungsträgern in der Gesundheitspolitik ist daher essenziell.

$\mathrm{P}$ rof. Dr. Matthias Augustin vom Universitätsklinikum HamburgEppendorf machte in seinem Vortrag auf drei Regeln aufmerksam:

1., ,Jederzeit und unerwartet können aus Gesundheitspolitik und Selbstverwaltung Maßnahmen kommen, die für unser Fach bedrohlich sind."

2. "Ohne Daten keine Argumentation!“

3. "Ohne Argumentation keine Wirkung!“

\section{Von der Selektivität der Daten zur} politischen Intervention

Die Forschung im Gesundheitswesen setzt sich aus drei Säulen zusammen: In der biomedizinischen Grundlagenforschung werden im experimentellen Ansatz erste Basiserkenntnisse gewonnen. In der klinischen Forschung erfolgen Studien, bei denen unter kontrollierten Bedingungen etwa ein Medikament seine Effektivität unter Beweis stellen muss. Die dritte Kategorie - die Versorgungsforschung - bezeichnete Augustin als ,Real-world-Beobachtung". Hier werden beispielsweise Beobachtungsstudien durchgeführt; auch öffentliches Gesundheitswesen, Epidemiologie oder Gesundheitsökonomie sind Themen der Versorgungsforschung.

Augustin wies auf die Selektivität von Daten hin, die z.B. von Krankenkassen, Apotheken, Arztpraxen, Krankenhäusern oder aus Umfragen stammen. Auch klinische Studien zeichnen sich durch selektive Daten aus: Von den Patienten, die etwa an der Langzeitbeobachtungsstudie RABBIT (Rheumatoide Arthritis: Beobachtung der Biologika-Therapie) teilnahmen, hätten in den Wirksamkeitsstudien für die entsprechenden Biologika nur zwischen 2333\% die Einschlusskriterien erfüllt.

Am Deutschen Netzwerk Versorgungsforschung sind allein mehr als 80 Fachgesellschaften und Verbände beteiligt. Die Rationale der Versorgungsforschung besteht zunächst in der Analyse der aktuellen Versorgungssituation, gefolgt vom Festlegen der Versorgungsziele und schließlich

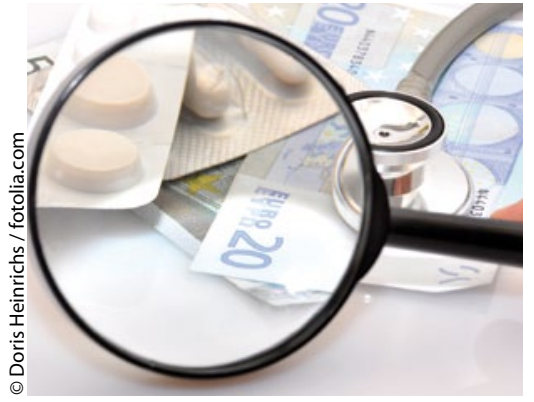

den notwendigen Konsequenzen für die zukünftige Gestaltung der Versorgung.

Als Beispiel für eine erfolgreiche Intervention - basierend auf Daten der Versorgungsforschung - nannte Augustin die Einflussnahme auf die umstrittene Entscheidung des Gemeinsamen Bundesausschusses, steroidhaltige topische Kombinationspräparate bei Hauterkrankungen von der Erstattung durch die Krankenkassen auszuschließen. Hier konnte mit Daten des Competenzzentrums Versorgungsforschung in der Dermatologie (CVderm) eine Rücknahme dieses Entschlusses erzielt werden..

Dr. Andreas Fischer

Augustin M. Warum Versorgungsforschung? 